\title{
Research on Fuzzy Multiple Attribute Route Evaluation Decision Method
}

\author{
Geng Yu, Qing Liu \\ Civil Aviation College, Shenyang Aerospace University, Shenyang, China \\ Email:13940298836@163.com
}

How to cite this paper: Yu, G. and Liu, Q. (2019) Research on Fuzzy Multiple Attribute Route Evaluation Decision Method. World Journal of Engineering and Technology, 7, 65-72.

https://doi.org/10.4236/wjet.2019.72B008

Received: May 5, 2019

Accepted: May 14, 2019

Published: May 17, 2019

\begin{abstract}
The route evaluation is an important basis for airlines to make route decisions. In order to solve the fuzzy multiple attribute route decision problem, route attribute weight was completely unknown, and the route evaluation fuzzy language information was given, the fuzzy language and preference information were converted into trapezoidal fuzzy numbers. A programming model was constructed by minimizing the total deviation between trapezoidal fuzzy numbers to determining the weight of each attribute in the route decision, By weighting the average of the route attribute value and the weights value, compared the expected value of fuzzy comprehensive evaluation for each route, and ranking alternatives by using the expected value operator of fuzzy variable. Finally, a numerical example was used to illustrate the proposed method. The results show that the method can be used by airlines to solve multiple attribute route decision problems with unknown weights, and the method is scientific and effective.
\end{abstract}

\section{Keywords}

Route Decision, Fuzzy Language, Multiple Attribute Decision Making, Trapezoidal Fuzzy Number, Expected Value

\section{Introduction}

The route is one of the elements of the airline passenger and cargo transportation market. The delivery strategy of the route capacity and the arrangement of the flight plan need to be based on the route. How to choose the route based on the existing capacity is a major business decision of the airline. It is an important task for airlines to improve the decision-making level of airlines by using scientific and effective methods to analyze and evaluate routes and select suitable routes to operate. Most domestic airlines still make decisions based on the current operating conditions of the routes to be selected. Therefore, people think of 
extracting the route target attributes first, and determining the attribute weights for route evaluation and analysis. Document [1] interpolated the target attribute, and the linear programming model was used to obtain the attribute weights and route sorting. Scientific planning of routes and rational use of airspace resources are the objectives of the air transport market [2].

In this paper, for fuzzy multi-attribute route decision problems with completely unknown attribute weights, the fuzzy evaluation language is transformed into trapezoidal fuzzy numbers. The weight of each attribute of the route is determined by the total deviation between trapezoidal fuzzy numbers. The weighted average method is used to adjust the fuzzy attribute values and weights. Integrate and sort the routes by comparing the fuzzy comprehensive evaluation of the expected values.

\section{Decision Principle and Method}

\subsection{Decision Principle}

The problem of route decision is actually a multi-objective planning problem. There are many factors affecting route decision. According to the literature [3], the five target attributes selected by the route analysis decision are as follows: The total short-term average passenger load factor, a profit attribute that reflects the operational efficiency of the route; The competitiveness, a profit attribute indicating the airline's ability to compete against other airlines on this route; The impact on the route network, a profit attribute representing the impact on future route operations and market size; The long-term market demand, a profit attributes related to local population size, economic status, etc; Cost fare ratio, a cost attribute that reflects the ratio of cost to fare level [3] [4]. In addition, Decision makers have different preferences for different routes due to special reasons such as policies. In practical problems, people usually use fuzzy language such as "good", "very good", "general" and "poor" to objectively evaluate and analyze the attributes of each route and provide preference information [5] [6]. According to the above principle, the route decision steps of this paper are in Figure 1.

\subsection{Decision Making Method}

In order to solve the fuzzy multiple attribute route decision problem, route attribute weight was completely unknown, and the route evaluation fuzzy language information was given, Let $X=\left\{x_{1}, x_{2}, \ldots x_{m}\right\}$ be $\mathrm{m}$ route sets, let $U=\left\{u_{1}, u_{2}, \ldots u_{n}\right\}$ be the route attribute set, and let $R=\left[r_{i j}\right]_{m \times n}$ be the route decision matrix. $r_{i j}$ represents the fuzzy language evaluation result of the route $X_{i}$ with respect to the route attribute $U_{j} . \quad r_{i j} \in M$, Let $M$ be a set of languages containing seven language values from low to high. $M=$ \{very low, low, lower, average, high, higher, very high $\}$. Let $T=\left\{t_{1}, t_{2}, \ldots t_{m}\right\}$ be the route preference vector, $t_{i} \in M$ represents the fuzzy language preference value of the decision maker on the route.

Using fuzzy numbers to quantify fuzzy languages is more accurate [7] [8] [9]. 


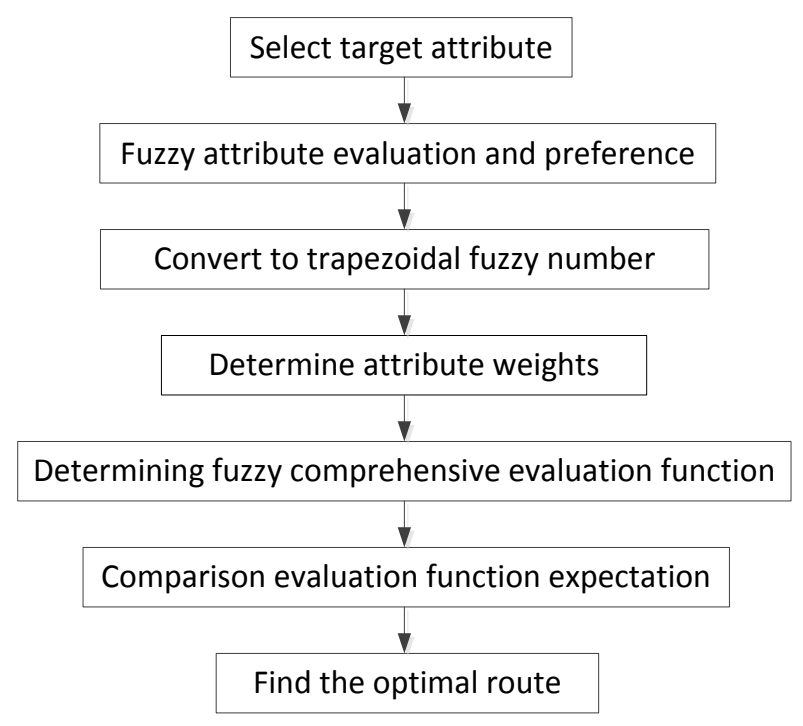

Figure 1. Route decision process.

Therefore, the trapezoidal fuzzy numbers corresponding to the income class attribute and the cost class attribute are as follows [4]:

The profit attribute: $\{$ very low $=(0,0,0,0.2)$; low $=(0,0,0.1,0.3)$; lower $=(0$, $0.2,0.2,0.4)$; general $=(0.3,0.5,0.5,1)$; higher $=(0.6,0.8,0.8,1)$; high $=(0.7,0.9$, $1,1)$; very high $=(0.8,1,1,1)\}$.

The cost attribute: $\{$ very low $=(0.8,1,1,1)$; low $=(0.7,0.9,1,1)$; lower $=(0.6$, $0.8,0.8,1)$; general $=(0.3,0.5,0.5,1)$; higher $=(0,0.2,0.2,0.4)$; high $=(0,0,0.1$, $0.3)$; very high $=(0,0,0,0.2)\}$

In this way, the route decision matrix $R=\left[r_{i j}\right]_{m \times n}$ and route preference vector $R=\left[r_{i j}\right]_{m \times n}$ given by fuzzy language are converted into trapezoidal fuzzy numbers. Expressed as fuzzy decision matrix $A=\left[\xi_{i j}\right]_{m \times n}$ and route preference vector $V=\left\{v_{1}, v_{2}, \ldots, v_{m}\right\} \quad[10]$.

Due to the different order of magnitudes between the attributes, the elements in $A=\left[\xi_{i j}\right]_{m \times n}$ are dimensionless attribute values. So convert $A=\left[\xi_{i j}\right]_{m \times n}$ to a normalization matrix $B=\left[\eta_{i j}\right]_{m \times n}$ [11]. In order to operate between the attributes, the equation is calculated as follows:

$$
\eta_{i j}=\frac{\xi_{i j}}{\sqrt{\sum_{i=1}^{m}\left[E\left(\xi_{i j}\right)\right]^{2}}} .
$$

Let the trapezoidal fuzzy variable be $\xi_{i j}=\left(r_{i j}{ }^{1}, r_{i j}{ }^{2}, r_{i j}{ }^{3}, r_{i j}{ }^{4}\right)$ and $\mathrm{E}$ be the expected value operator. Then the expected value of $\xi_{i j}$ is

$$
E\left(\xi_{i j}\right)=\frac{1}{4}\left(r_{i j}{ }^{1}, r_{i j}{ }^{2}, r_{i j}{ }^{3}, r_{i j}{ }^{4}\right)
$$

Let the attribute weight vector be $\omega=\left\{\omega_{1}, \omega_{2}, \ldots, \omega_{n}\right\}$, and $\omega_{j}$ is an unknown variable that satisfies $\sum_{i=1}^{n} \omega_{j}=1, \omega_{j} \geq 0$. Integrate normalized route decision matrices and attribute weight vectors with weighted average rule. The fuzzy 
comprehensive evaluation function for each route is

$$
f_{i}=\sum_{i=1}^{n} \eta_{i j} \cdot \omega_{i j}, i=1,2, \ldots, m
$$

In order to balance both subjective and objective evaluations, the total deviation between attribute values and preference values is kept as small as possible. Determine the weight of each attribute. Since the absolute value of the difference between the expected values of the two trapezoidal fuzzy numbers can be used to indicate their degree of deviation [12]. The quadratic programming model is constructed as follows:

$$
\begin{aligned}
& \min F(\omega)=\sum_{j=1}^{n} \sum_{i=1}^{m}\left(E\left(\eta_{i j}\right)-E\left(v_{i j}\right)\right)^{2} \cdot \omega_{j}{ }^{2} \\
& \text { s.t. } \sum_{j=1}^{n} \omega_{j}=1 \\
& \omega_{j} \geq 0, j=1,2, \ldots, n
\end{aligned}
$$

$F(\omega)$ represents the total deviation of the decision maker's subjective preference value from the objective evaluation value.

Model (4) is a quadratic programming on weights $\omega_{j}, j=1,2, \ldots, n$, constructing a Lagrangian function:

$$
L(\omega, \lambda)=\sum_{j=1}^{n} \sum_{i=1}^{m}\left[E\left(\eta_{i j}\right)-E\left(v_{i}\right)\right]^{2} \omega_{j}^{2}+2 \lambda\left(\sum_{j=1}^{n} \omega_{j}-1\right)
$$

Solve the following partial derivative equations:

$$
L \omega_{j}=2 \sum_{i=1}^{m}\left[E\left(\eta_{i j}\right)-E\left(v_{i}\right)\right]^{2} \cdot \omega_{j}+2 \lambda=0, j=1,2, \ldots, n
$$

The weighting formula is as follows:

$$
\omega_{j}=\frac{\frac{1}{\sum_{j=1}^{n} \frac{1}{\sum_{i=1}^{m}\left[E\left(\eta_{i j}\right)-E\left(v_{i}\right)\right]^{2}}}}{\sum_{i=1}^{m}\left[E\left(\eta_{i j}\right)-E\left(v_{i}\right)\right]^{2}}, j=1,2, \ldots, n
$$

Substituting the weights $\omega_{j}, j=1,2, \ldots, n$ obtained by Equation (7) into Equation (3). Find the fuzzy comprehensive evaluation value $f_{i}$ of each route, calculating $E\left[f_{i}\right], i=1,2, \ldots, m$, and sorting them by size to get the corresponding sorting for each route. The route decision method is as follows:

Step 1: Constructing a fuzzy language decision matrix based on the information given by experts, and converting the fuzzy language of each route attribute into a trapezoidal fuzzy number. Find the decision matrix $A=\left[\xi_{i j}\right]_{m \times n}$ and the route preference vector $V=\left(v_{1}, v_{2}, \ldots, v_{m}\right)$.

Step 2: Convert the decision matrix $A=\left[\xi_{i j}\right]_{m \times n}$ into a normalized decision matrix $B=\left[\eta_{i j}\right]_{m \times n}$ according to Formula (1).

Step 3: Find each attribute $\omega_{j}, j=1,2, \ldots, n$ weight by Formula (2) (7). 
Step 4: Obtain the fuzzy comprehensive evaluation value of each route according to Formula (3).

Step 5: Calculate and sort them by size to select the optimal route.

\section{Example Analysis}

According to the survey, an airline will evaluate and evaluate four routes in the Northeast. Experts are invited to conduct fuzzy language assessments on these four routes. The five target attributes are selected as follows: the total short-term average passenger load factor of the route; the competitiveness; impact on the route network; the long-term market demand; Cost fare ratio; The decision maker also gives preference information on the four routes to be selected. The expert evaluation results and route preference information of these four routes are shown in Table 1.

Sort and optimize these four routes using the above method.

1) Convert fuzzy language into trapezoidal fuzzy number to get decision matrix $A=\left(A_{1} A_{2} A_{3} A_{4} A_{5}\right)$.

The decision matrix after the conversion of the five target attributes is as follows:

Average passenger load factor $u 1: \quad A_{1}=\left(\begin{array}{llll}0.0 & 0.2 & 0.2 & 0.4 \\ 0.3 & 0.5 & 0.5 & 0.7 \\ 0.0 & 0.0 & 0.1 & 0.3 \\ 0.0 & 0.2 & 0.2 & 0.4\end{array}\right)$

Competitiveness $u 2: \quad A_{2}=\left(\begin{array}{cccc}0.6 & 0.8 & 0.8 & 1.0 \\ 0.8 & 1.0 & 1.0 & 1.0 \\ 0.3 & 0.5 & 0.5 & 0.7 \\ 0.6 & 0.8 & 0.8 & 1.0\end{array}\right)$

Impact on the route network $u 3: \quad A_{3}=\left(\begin{array}{llll}0.7 & 0.9 & 1.0 & 1.0 \\ 0.6 & 0.8 & 0.8 & 1.0 \\ 0.6 & 0.6 & 0.8 & 1.0 \\ 0.3 & 0.5 & 0.5 & 0.7\end{array}\right)$

Long-term market demand $u 4: A_{4}=\left(\begin{array}{cccc}0.7 & 0.9 & 1.0 & 1.0 \\ 0.7 & 0.9 & 1.0 & 1.0 \\ 0.6 & 0.8 & 0.8 & 1.0 \\ 0.6 & 0.8 & 0.8 & 1.0\end{array}\right)$

Cost fare ratio $u 5: \quad A_{5}=\left(\begin{array}{llll}0.8 & 1.0 & 1.0 & 1.0 \\ 0.7 & 0.9 & 1.0 & 1.0 \\ 0.8 & 1.0 & 1.0 & 1.0 \\ 0.7 & 0.9 & 1.0 & 1.0\end{array}\right)$

The route preference vector is as follows:

$$
\begin{aligned}
V= & \left(\left(\begin{array}{llll}
0.6 & 0.8 & 0.8 & 1.0
\end{array}\right)\left(\begin{array}{llll}
0.8 & 1.0 & 1.0 & 1.0
\end{array}\right)\right. \\
& \left(\begin{array}{llllll}
0.7 & 0.9 & 1.0 & 1.0
\end{array}\right)\left(\begin{array}{llll}
0.7 & 0.9 & 1.0 & 1.0
\end{array}\right)
\end{aligned}
$$

2) Converting the decision matrices $A$ of five attributes into standardized decision matrices $B$. 
Table 1. Comparison of route fuzzy language assessment.

\begin{tabular}{ccccc}
\hline & $x 1$ & $x 2$ & $x 3$ & $x 4$ \\
\hline Average passenger load factor $u 1$ & lower & general & low & lower \\
Competitiveness $u 2$ & higher & very high & general & higher \\
Impact on the route network $u 3$ & high & higher & higher & general \\
Long-term market demand $u 4$ & high & high & higher & higher \\
Cost fare ratio $u 5$ & very low & low & very low & low \\
Preference information $v$ & More willing & very willing to & willing & willing \\
\hline
\end{tabular}

$$
\begin{aligned}
B & =\left(B_{1} B_{2} B_{3} B_{4} B_{5}\right) \\
B_{1} & =\left(\begin{array}{llll}
0.000 & 0.343 & 0.343 & 0.686 \\
0.515 & 0.858 & 0.858 & 1.201 \\
0.000 & 0.000 & 0.172 & 0.515 \\
0.000 & 0.343 & 0.343 & 0.686
\end{array}\right) \\
B_{2} & =\left(\begin{array}{llll}
0.385 & 0.513 & 0.513 & 0.641 \\
0.513 & 0.641 & 0.641 & 0.641 \\
0.192 & 0.321 & 0.321 & 0.449 \\
0.385 & 0.513 & 0.513 & 0.641
\end{array}\right) \\
B_{3} & =\left(\begin{array}{llll}
0.458 & 0.588 & 0.654 & 0.654 \\
0.392 & 0.523 & 0.523 & 0.654 \\
0.392 & 0.392 & 0.523 & 0.654 \\
0.196 & 0.327 & 0.327 & 0.458
\end{array}\right) \\
B_{4} & =\left(\begin{array}{llll}
0.411 & 0.528 & 0.587 & 0.587 \\
0.411 & 0.528 & 0.587 & 0.587 \\
0.352 & 0.470 & 0.470 & 0.587 \\
0.352 & 0.470 & 0.470 & 0.587
\end{array}\right) \\
B_{5} & =\left(\begin{array}{llll}
0.432 & 0.540 & 0.540 & 0.540 \\
0.378 & 0.486 & 0.540 & 0.540 \\
0.432 & 0.540 & 0.540 & 0.540 \\
0.378 & 0.486 & 0.540 & 0.540
\end{array}\right)
\end{aligned}
$$

3) The weight values of the attributes determined by Equations (2) and (7) are:

$$
\omega_{1}=0.135, \omega_{2}=0.209, \omega_{3}=0.197, \omega_{4}=0.229, \omega_{5}=0.230 .
$$

4) The fuzzy comprehensive evaluation values of the four routes obtained by Formula (3) are trapezoidal fuzzy numbers:

$$
\begin{aligned}
& f_{1}=(0.364,0.514,0.541,0.602) \\
& f_{2}=(0.505,0.586,0.611,0.684) \\
& f_{3}=(0.297,0.376,0.425,0.551) \\
& f_{4}=(0.287,0.463,0.450,0.575)
\end{aligned}
$$

Calculate the expected values of the fuzzy comprehensive evaluation values of the four routes: 


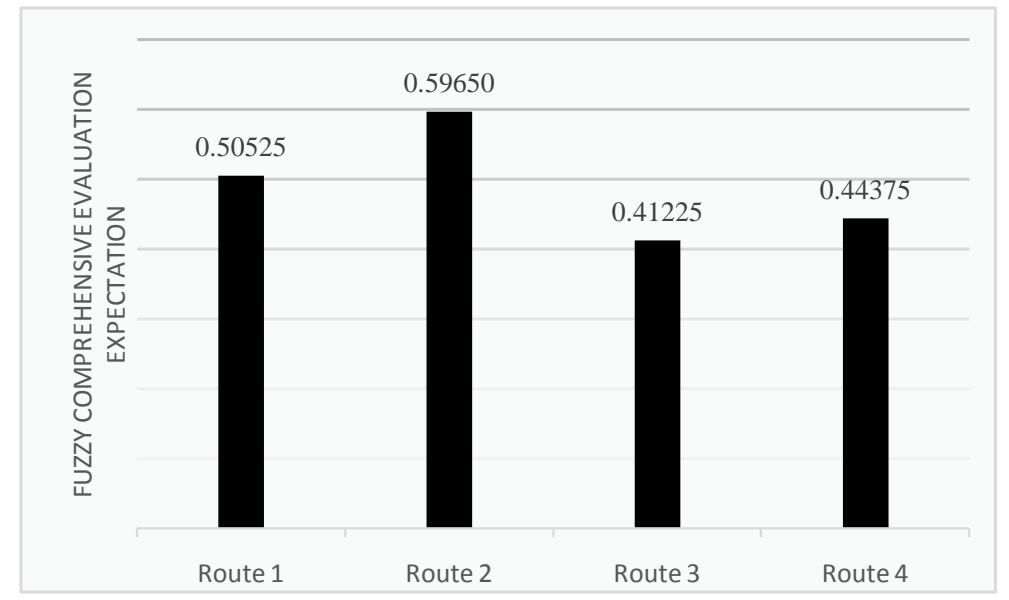

Figure 2. Comparison of comprehensive evaluation of routes.

$$
E\left(f_{1}\right)=0.50525, E\left(f_{2}\right)=0.59650, E\left(f_{3}\right)=0.41225, E\left(f_{4}\right)=0.44375
$$

According to the size order, the order of the four routes is route $2>$ route $1>$ route $4>$ route 3 , and the optimal route is route 2 . The result is shown (Figure 2).

\section{Summary}

The solution to the fuzzy multiple attribute route decision problem is proposed in this paper. The total deviation between the trapezoidal fuzzy numbers is used to determine the weight of each attribute of the route, and the expected value of the fuzzy comprehensive evaluation is compared to sort and optimize the scheme. The method is scientific and effective, and helps airlines to make route analysis decisions.

\section{Acknowledgements}

The authors are thankful to the Aeronautical Operation Technology Laboratory of Shenyang Aerospace University for providing the necessary facilities and financial support for the investigation. Thanks to Wang Han and Hao Jun for their help and guidance.

\section{Conflicts of Interest}

The authors declare no conflicts of interest regarding the publication of this paper.

\section{References}

[1] Bai, M.G. and Zhu, J.F. (2005) A Method of Airline Route Decision. Statistics and decision, 20, 151-153.

[2] Yu, J., Sun, J.H. and Zhu, X.H. (2007) Imagination of National Route Network Planning and Construction. Comprehensive Transportation, 12, 37-40.

[3] Qin, H.X. and Cheng, X.J. (2000) A Systematical Study on Decision-Making of Se- 
lecting Routes for Airlines. Operations Research and Management Science, 3, 56-63.

[4] Bai, M.G. (2006) Research on Optimization Design Problems of Airline Network. Nanjing Aerospace University, Nanjing.

[5] Ma, S.L. and Liu, X.H. (2009) Fuzzy Multiple Attribute Decision Making Method With Preference Information on Alternatives in Investment Decision Based on Fuzzy Linguistic Assessment. Journal of Tangshan Teachers College, 31, 39-41.

[6] Zeng, L. (2004) Fuzzy Multiple Attribute Decision Making Method with Preference Information on Alternatives Based on Fuzzy Linguistic Assessments. Proceedings of the 12 th National Conference on Fuzzy Systems and Fuzzy Mathematics, Hunan, 267-270.

[7] Lv, Z.Y. (2014) Research Fuzzy Multiple Attribute Decision Making and Its Application. Southwest Jiaotong University, Chengdu.

[8] Gao, Y. (2010) A Study on the Method of Multiple Attributes Decision Making Based on Fuzzy Decision Making Matrix. Nanjing Aerospace University, Nanjing.

[9] Li, M.H. and Xia, J.B. (2010) Survey on Weight Information Problem Based on FN-E\&DM. Computer Science, 10, 23-26.

[10] Shi, Y.Y., Xu, Y.Y. and Luo, S.Y. (2017) An Approach of Hesitant Intuitionistic Fuzzy Linguistic Grey Relational Decision Making Based on Combination Weight. Journal of Science of Teachers' College and University, 1, 11-14.

[11] Liu, F. (2014) Research on Risk Investment Project Selection Method Based on Hybrid Multi-Attribute Group Decision Making. Jiangxi University of Finance and Economics, Nanchang.

[12] Zeng, S.Y. and Long, J. (2009) Fuzzy Multiple Attribute Decision Making Method Based on The Maximal Deviation. Journal of Huaihua University, 5, 16-19. 